\title{
Impact of pressure load caused by right ventricular outflow tract obstruction on right ventricular volume overload in patients with repaired tetralogy of Fallot
}

\author{
Byung Won Yoo, MD, ${ }^{\mathrm{a}}$ Jung Ok Kim, MD, ${ }^{\mathrm{a}}$ Young Jin Kim, MD, ${ }^{\mathrm{b}}$ Jae Young Choi, MD, ${ }^{\mathrm{a}}$ \\ Han Ki Park, MD, ${ }^{\mathrm{c}}$ Young Hwan Park, MD, ${ }^{\mathrm{c}}$ and Jun Hee Sul, $\mathrm{MD}^{\mathrm{a}}$
}

\begin{abstract}
Objectives: In correction of tetralogy of Fallot (TOF), surgical strategies to minimize right ventricular outflow tract (RVOT) enlargement have recently been preferred. However, we may be confronted with residual pulmonary stenosis (PS) combined with pulmonary regurgitation (PR), and how the pressure load affects these patients is not evident.
\end{abstract}

\begin{abstract}
Methods: We compared 51 patients with PR and significant PS (PR with PS group) with 87 patients with PR without significant PS (PR group) using echocardiography and cardiac magnetic imaging. We evaluated the differences in parameters derived by magnetic resonance imaging between the 2 groups and the influence of the pressure load on right ventricular (RV) volume and function.

Results: Although the PR fraction was similar between the 2 groups, the PR with PS group showed significantly smaller RV end-diastolic volume $\left(136.7 \pm 26.5 \mathrm{~mL} / \mathrm{m}^{2}\right.$ vs $\left.151.2 \pm 34.7 \mathrm{~mL} / \mathrm{m}^{2} ; P=.01\right)$, RV end-systolic volume $\left(68.1 \pm 23.7 \mathrm{~mL} / \mathrm{m}^{2}\right.$ vs $\left.80.2 \pm 27.5 \mathrm{~mL} / \mathrm{m}^{2} ; P=.01\right)$, and slightly better RV ejection fraction $(51.1 \% \pm$ $9.8 \%$ vs $47.6 \% \pm 8.9 \% ; P=.03)$ than the PR group. For influence of the pressure load, PR fraction $(r=-0.18$, $P=.03)$, RV end-diastolic volume ( $r=-0.25, P=.003)$, and RV end-systolic volume $(r=-0.24, P=.005)$ were decreased as peak pressure gradient of PS was higher. Linear regression analysis revealed that both PR fraction and peak pressure gradient of PS were independent predictors for RV volume.
\end{abstract}

Conclusions: Our study demonstrated that the RV pressure load prevented RV dilatation from chronic PR without systolic dysfunction. It is suggested that a proper relief of RVOT obstruction with acceptable residual stenosis is more advantageous than aggressive RVOT enlargement in the long-term outcome of repaired TOF. (J Thorac Cardiovasc Surg 2012;143:1299-304)

Earn CME credits at

http://cme.ctsnetjournals.org

Although total correction of tetralogy of Fallot (TOF) has an excellent outcome, it leaves a varying degree of pulmonary regurgitation (PR) in the majority of patients. It is widely known that chronic PR can lead to right ventricular (RV) volume overload and dysfunction, resulting in increase of long-term morbidity and mortality. ${ }^{1-4}$ However, deterioration of $\mathrm{RV}$ function caused by $\mathrm{PR}$ is not

\footnotetext{
From the Division of Pediatric Cardiology, ${ }^{\mathrm{a}}$ Department of Radiology, ${ }^{\mathrm{b}}$ and Department of Cardiovascular Surgery, ${ }^{\mathrm{c}}$ Yonsei University College of Medicine, Seoul, Korea.

Disclosures: Authors have nothing to disclose with regard to commercial support.

Received for publication Aug 4, 2011; revisions received Nov 18, 2011; accepted for publication Dec 14, 2011; available ahead of print Jan 13, 2012.

Address for reprints: Byung Won Yoo, MD, Division of Pediatric Cardiology, Cardiovascular Center, Yonsei University College of Medicine, 134 Shinchon-dong, Seodaemun-gu, Yonsei University Health System, Seoul, 120-752, Korea (E-mail: bwyoo@yuhs.ac).

$0022-5223 / \$ 36.00$

Copyright (c) 2012 by The American Association for Thoracic Surgery doi:10.1016/j.jtcvs.2011.12.033
}

consistent in the patients. Some patients have shown severely progressive RV dilatation and dysfunction as time went by, whereas other patients have tolerated PR well, resulting in preserved RV volume and function. Under this circumstance, many previous studies have tried to determine the factors related to RV volume overload in patients with repaired TOF. Some of them have revealed that palliative shunt operation, age at repair, use of transannular patch, and aneurysmal dilatation of the RV outflow tract have some adverse effects. ${ }^{4-10}$ However, it is not always easy to demonstrate a clear impact of the related factors on RV mechanics owing to compounding interactions among various factors.

Recently, most centers follow the surgical strategies to reduce pulmonary valvectomy or transannular patch rate to prevent aggravated PR with deleterious effects on longterm morbidity. However, we may be confronted with residual pulmonary stenosis (PS) combined with PR, and how the pressure load affects these patients is not evident.

The purpose of this study is to evaluate whether the pressure load by residual PS has any influence on RV volume and function after repair of TOF and whether the residual PS resulting from minimal RV outflow tract (RVOT) enlargement strategies is acceptable. 


$$
\begin{aligned}
& \text { Abbreviations and Acronyms } \\
& \mathrm{LV} \quad=\text { left ventricle (ventricular) } \\
& \text { MR = magnetic resonance } \\
& \text { MRI = magnetic resonance imaging } \\
& \mathrm{PR}=\text { pulmonary regurgitation } \\
& \text { PS = pulmonary stenosis } \\
& \text { PSPG = peak pressure gradient of residual } \\
& \text { pulmonary stenosis } \\
& \text { PVR = pulmonary valve replacement } \\
& \mathrm{RV} \quad=\text { right ventricle (ventricular) } \\
& \text { RVESVi }=\text { right ventricular end-systolic volume } \\
& \text { index } \\
& \text { RVEDVi }=\text { right ventricular end-diastolic volume } \\
& \text { index } \\
& \text { RVOT = right ventricular outflow tract } \\
& \text { TOF }=\text { tetralogy of Fallot }
\end{aligned}
$$

\section{METHODS}

\section{Subject}

We retrospectively reviewed the case histories of 190 patients with repaired TOF who received cardiac magnetic resonance imaging (MRI) at Yonsei Cardiovascular Center from July 2004 to June 2009. In our policy, if the patients with repaired TOF show considerable RV enlargement with significant PR, we routinely perform an MRI examination. Our present study retrospectively reviewed those data. An echocardiogram was taken in all patients within 3 months before or after the cardiac MRI study and was reviewed by a single investigator. The patients who had significant regurgitation of valves other than the pulmonary valve and residual intracardiac shunts were excluded. Patients were assigned to the PR with PS group if they had residual PS with their echocardiogram showing the sum of the Doppler peak pressure gradient of more than $25 \mathrm{~mm} \mathrm{Hg}$ at the infundibular and valvular and supravalvular levels. Patients were assigned to the PR group if they had no significant PS with their echocardiogram showing a Doppler peak pressure gradient less than $25 \mathrm{~mm} \mathrm{Hg}$ at the aforementioned sites. Patients were excluded if they had significant unilateral branch PS with a Doppler gradient of more than $10 \mathrm{~mm} \mathrm{Hg}$. RV systolic pressure was estimated by using the modified Bernoulli equation (RV systolic pressure $=4 \cdot$ [tricuspid regurgitation jet velocity $]^{2}+$ estimated right atrial pressure). Restrictive physiology of the RV was defined as the presence of antegrade diastolic flow, demonstrable by pulsed wave Doppler echocardiography in the distal pulmonary artery throughout respiration. ${ }^{11}$

This retrospective study was approved by the Institutional Review Board of Severance Hospital (Seoul, Korea).

\section{Cardiac MRI}

Our study was performed with a commercially available 1.5-T magnetic resonance (MR) scanner (Intera Achieva; Philips Medical Systems, Best, the Netherlands). Both ventricular volume and function were assessed with an electrocardiogram-gated steady state free-precession cine MR pulse sequence during breath-holding periods. We used a stack of 10 to 12 slices with 25 phases/cycle at 8- to 10 -mm thickness in shortaxis planes. All volumes were indexed for the body surface area of each patient.

Flow measurements were performed in the proximal main pulmonary artery and both pulmonary arteries with the use of a velocity-encoded phase contrast sequence. Forward and backward flows were calculated at the proximal main pulmonary artery and each individual pulmonary artery, allowing us to derive the PR fraction.

A single investigator analyzed MRIs using the dedicated software (ViewForum, version 4.1; Philips Medical Systems).

\section{Statistics}

Results are expressed as mean \pm 1 standard deviation for the normal distribution data. Patient clinical data and MRI parameters between the 2 groups were compared using the Student $t$ test for continuous data and the Pearson $\chi^{2}$ test for nonnumeric parameters. The strength of relationship among MRI parameters in each group was tested using the Pearson correlation. For identification of independent predictors for RV volume and function, linear regression analysis was used. The clinical parameters (sex, age at study, age at TOF repair, follow-up duration from TOF repair, palliative shunt history, transannular patch history), echocardiographic parameters (the presence of RV restrictive physiology, the peak pressure gradient of residual PS [PSPG]), and MRI parameters (PR fraction, RV volume indexes [in analysis for RV ejection fraction]) were analyzed in a univariate model, and all parameters that showed statistical significance in a univariate model were included in multivariate model analysis. SPSS for Windows (version 12.0; SPSS Inc, Chicago, Ill) was used for data analysis.

\section{RESULTS}

\section{Study Population}

The characteristics and clinical data for subjects are shown in Table 1. The PR group was composed of 87 patients and the PR with PS group was composed of 51 patients. There was no significant difference between the 2 groups in terms of age and follow-up duration after total correction.

The patients in the PR with PS group had more previous palliative shunt histories than the patients in the PR group $(27.5 \%$ vs $12.6 \% ; P=.02)$. However, there was no significant difference in age at total correction (PR group, $2.1 \pm$ 2.4 years; PR with PS group, $3.1 \pm 3.7$ years) and the numbers of patients with transannular patch (PR group, 65 [74.7\%]; PR with PS group, 41 [80.4\%]). For detailed surgical techniques, in the PR with PS group, intraoperative $\mathrm{RV}$ pressure (PR group, $43.0 \pm 11.5 \mathrm{~mm} \mathrm{Hg}$; PR with PS group, $50.3 \pm 15.2 \mathrm{~mm} \mathrm{Hg} ; P=.025)$ and right ventricular/left ventricular (RV/LV) pressure ratio (PR group, 0.42 \pm 0.12 ; PR with PS group, $0.51 \pm 0.14 ; P=.007$ ) were higher than those in the PR group. However, the frequency of pulmonary valvotomy or pulmonary valvectomy and RVOT size after relief of RVOT obstruction were not different between the 2 groups.

The PSPG was $12.8 \pm 7.0 \mathrm{~mm} \mathrm{Hg}$ in the PR group and $34.2 \pm 10.0 \mathrm{~mm} \mathrm{Hg}$ in the PR with PS group. The frequency of restrictive physiology of the RV was slightly more in the PR with PS group (42\%) than in the PR group (36\%) without statistical significance.

\section{Differences in RV Volume and Function}

The result of our MRI study showed that although PR fraction was similar between the 2 groups $(40.8 \% \pm$ $10.0 \%$ for the PR group; $42.7 \% \pm 11.5 \%$ for the PR 
TABLE 1. Characteristics and clinical data of subjects

\begin{tabular}{|c|c|c|c|}
\hline & PR group $(n=87)$ & PR with PS group $(n=51)$ & $P$ value \\
\hline $\operatorname{Sex}(M: F)$ & $56: 30$ & $30: 21$ & .46 \\
\hline Age (y) & $16.6 \pm 6.6$ & $17.5 \pm 8.3$ & .52 \\
\hline $\operatorname{BSA}\left(\mathrm{m}^{2}\right)$ & $1.45 \pm 0.35$ & $1.45 \pm 0.28$ & .99 \\
\hline Age at repair $(y)$ & $2.1 \pm 2.4$ & $3.1 \pm 3.7$ & .08 \\
\hline Follow-up after repair (y) & $14.4 \pm 5.2$ & $14.4 \pm 6.0$ & .97 \\
\hline QRS duration $(\mathrm{ms})$ & $144.0 \pm 25.5$ & $134.6 \pm 31.1$ & .06 \\
\hline Palliative shunt & $11(12.6 \%)$ & $14(27.5 \%)$ & .03 \\
\hline Transannular patch & $65(74.7 \%)$ & $41(80.4 \%)$ & .42 \\
\hline Pulmonary valvotomy* & $41 / 72(56.9 \%)$ & $21 / 41(51.2 \%)$ & .42 \\
\hline Pulmonary valvectomy* & $25 / 72(34.7 \%)$ & $15 / 41(36.6 \%)$ & .96 \\
\hline RVOT size after enlargement* $(\mathrm{mm})$ & $11.9 \pm 1.9$ & $12.5 \pm 1.7$ & .12 \\
\hline Intraop RV pressure* (mm Hg) & $43.0 \pm 11.5$ & $50.3 \pm 15.2$ & .025 \\
\hline Intraop RV/LV pressure* $(\mathrm{mm} \mathrm{Hg})$ & $0.42 \pm 0.12$ & $0.51 \pm 0.14$ & .007 \\
\hline PSPG (mm Hg) & $12.8 \pm 7.0$ & $34.2 \pm 10.0$ & $<.001$ \\
\hline Estimated RV pressure $†(\mathrm{~mm} \mathrm{Hg})$ & $36.4 \pm 5.3$ & $59.6 \pm 9.9$ & $<0.001$ \\
\hline Restrictive physiology of RV & $31(35.6 \%)$ & $21(41.1 \%)$ & .55 \\
\hline
\end{tabular}

$P R$, Pulmonary regurgitation; $P S$, pulmonary stenosis; $M$, male; $F$, female; $B S A$, body surface area; $R V O T$, right ventricular outflow tract; Intraop, intraoperative pressure data after RVOT enlargement; $R V$, right ventricular; $L V$, left ventricular; $P S P G$, peak pressure gradient of residual pulmonary stenosis. *Detailed surgical techniques and intraoperative data were investigated in patients with full operation record (72 in PR group, 41 in PR with PS group). $\nmid R V$ pressure estimated by tricuspid regurgitation jet velocity using modified Bernoulli equation.

with PS group; $P=.3$ ), the PR with PS group showed significantly smaller RV end-diastolic volume index (RVEDVi, $136.7 \pm 26.5 \mathrm{~mL} / \mathrm{m}^{2}$ vs $151.2 \pm 34.7 \mathrm{~mL} / \mathrm{m}^{2}$ $P=.01)$ and RV end-systolic volume index (RVESVi, $68.1 \pm 23.7 \mathrm{~mL} / \mathrm{m}^{2}$ vs $80.2 \pm 27.5 \mathrm{~mL} / \mathrm{m}^{2} ; P=.01$ ) than the PR group (Table 2, Figure 1). Furthermore, RV ejection fraction was slightly higher in the PR with PS group than in the PR group $(51.2 \% \pm 9.9 \%$ vs $47.6 \%$ $\pm 8.9 \% ; P=.03$ ).

\section{Relationships Between Cardiac MRI-derived Parameters}

In correlation analysis, RV volume indexes were increased according to the severity of PR with a tight relationship (RVEDVi: $r=0.53, P<.001$; RVESVi: $r=0.43$, $P<.001$ ), and RV function decreased in proportion to the RV volume indexes (RVEDVi: $r=-0.46, P<.001$; RVESVi: $r=-0.79, P<.001)$. PR was not correlated with the RV ejection fraction $(r=-0.15, P=.08)$.
RV volume overload was related to LV volume and function. As the RV volume indexes increased, LV end-diastolic volume index increased (RVEDVi: $r=0.33, P<.001$; RVESVi: $r=.29, P=.001)$ and, consequently, LV ejection fraction was decreased (RVEDVi: $r=-0.37, P<.001$; RVESVi: $r=-0.49, P<.001)$. Also, both ventricular functions were closely correlated with each other $(r=0.5$, $P<.001)$. In each subgroup, the relationships among the PR fraction and both ventricular volume and function were same as those of the total subjects.

\section{Impact of Pressure Load on RV Volume and Function}

The analysis of the influence of pressure load showed that as PSPG was higher, PR fraction $(r=-0.18, P=.03)$, RVEDVi $(r=-0.25, P=.003)$, and RVESVi $(r=-0.24$, $P=.005$ ) decreased (Figure 2$). \mathrm{RV}$ ejection fraction was not affected $(r=0.1, P=.2)$.

In subgroup analysis, PSPG was not correlated with PR fraction in the PR group, but was more inversely correlated

TABLE 2. Hemodynamic data from cardiac magnetic resonance imaging

\begin{tabular}{lccc}
\hline & PR group $(\mathbf{n}=\mathbf{8 7})$ & PR with PS group $(\mathbf{n}=\mathbf{5 1})$ & P value \\
\hline Pulmonary regurgitation fraction $(\%)$ & $42.7 \pm 11.5$ & $40.8 \pm 10.0$ & .33 \\
RV end-diastolic volume $\left(\mathrm{mL} / \mathrm{m}^{2}\right)$ & $151.2 \pm 34.7$ & $136.7 \pm 26.5$ & .01 \\
RV end-systolic volume $\left(\mathrm{mL} / \mathrm{m}^{2}\right)$ & $80.2 \pm 27.5$ & $68.1 \pm 23.7$ & .01 \\
RV stroke volume $\left(\mathrm{mL} / \mathrm{m}^{2}\right)$ & $71.3 \pm 15.3$ & $68.5 \pm 8.3$ & .29 \\
RV ejection fraction $(\%)$ & $47.6 \pm 8.9$ & $51.2 \pm 9.9$ & .03 \\
LV end-diastolic volume $\left(\mathrm{mL} / \mathrm{m}^{2}\right)$ & $78.6 \pm 20.7$ & $79.4 \pm 17.5$ & .83 \\
LV end-systolic volume $\left(\mathrm{ml} / \mathrm{m}^{2}\right)$ & $32.6 \pm 9.8$ & $32.5 \pm 10.9$ & .99 \\
LV stroke volume $\left(\mathrm{mL} / \mathrm{m}^{2}\right)$ & $46.2 \pm 7.6$ & $47.7 \pm 8.3$ & .28 \\
LV ejection fraction $(\%)$ & $58.6 \pm 8.0$ & $59.9 \pm 6.3$ & .32 \\
\hline
\end{tabular}

$P R$, Pulmonary regurgitation; $P S$, pulmonary stenosis; $R V$, right ventricular; $L V$, left ventricular. 


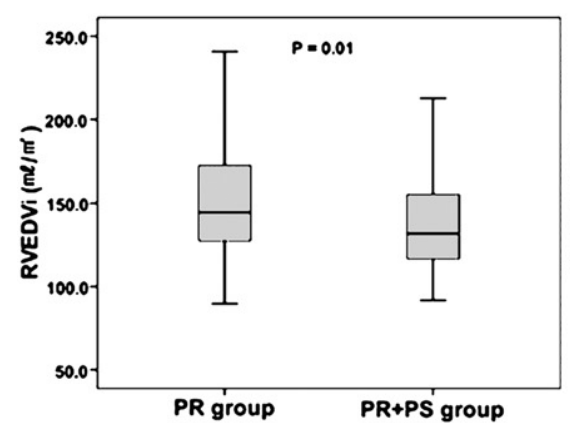

A

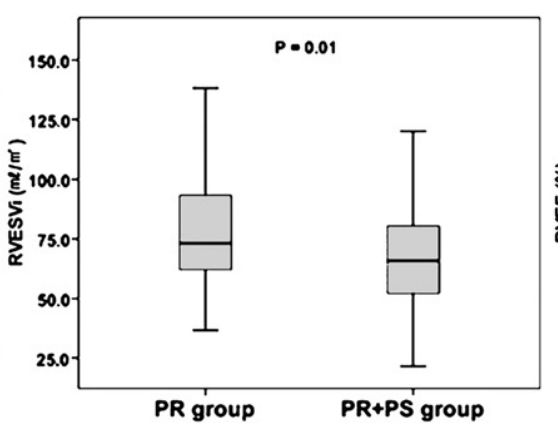

B

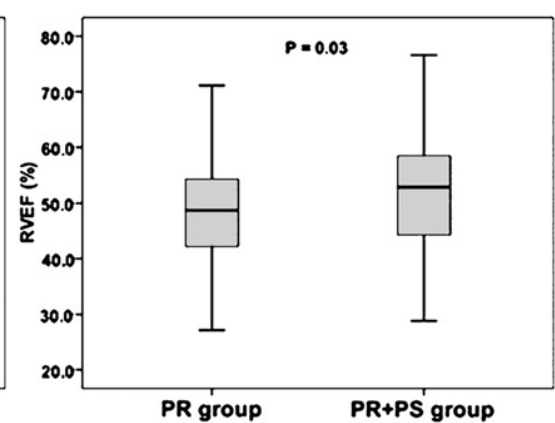

C

FIGURE 1. Comparisons of right ventricular volume and function between the pulmonary regurgitation (PR) group and the pulmonary regurgitation with pulmonary stenosis $(P R+P S)$ group. A, Right ventricular end-diastolic volume indexes $(R V E D V i)$, (B) right ventricular end-systolic volume indexes $(R V E S V i)$, and $(\mathrm{C})$ right ventricular ejection fraction $(R V E F)$.

in the PR with PS group $(r=-0.31, P=.03)$. Both groups revealed that RV pressure load was not correlated with RV dysfunction (PR group: $r=0.12, P=.3$; PR with PS group: $r=0.07, P=.6$ ).

\section{Predictors for RV Volume and Function}

In univariate analysis, only 2 variables, PR fraction and PSPG, were revealed as predictors of RV volume: PR fraction $(\beta=1.58, P<.001)$ and PSPG $(\beta=-0.55, P=.002)$ for RVEDVi; PR fraction $(\beta=1.04, P<.001)$ and PSPG $(\beta=-0.49, P=.005)$ for RVESVi. As predictors of RV ejection fraction, $\mathrm{RV}$ volume indexes were confirmed (RVEDVi: $\beta=-0.13, P<.001$; RVESVi: $\beta=-0.28$, $P<.001)$ whereas PSPG did not reach the statistical significance $(\beta=0.12, P=.053)$. The results of multivariate analysis are showed in Table 3.

\section{DISCUSSION}

Our study demonstrated that patients with residual PS had less RV volume overload and no deterioration of RV function compared with patients without PS even if both groups had similarly severe PR. Furthermore, pressure gradient of PS had a statistically significant countercorrelation with PR fraction, RVEDVi, and RVESVi, indicating that RV pressure load prevented RV dilatation from chronic PR. This result supports why minimal RVOT enlargement strategies should be chosen despite residual PS.

In a previous study, Chaturvedi and colleagues ${ }^{12}$ showed that transient balloon occlusion of a unilateral branch pulmonary artery increased both RV pressure and PR. After this study, residual PS has long been recognized as a risk factor that has an adverse effect on PR. However, a recent study to evaluate the risk factors for early pulmonary valve replacement (PVR) in repaired TOF demonstrated that as the smallest size of branch pulmonary artery decreased, the interval to PVR increased. ${ }^{13}$ We thought that the previous study by Chaturvedi's group ${ }^{12}$ should be carefully interpreted because transient balloon occlusion causes abrupt asymmetric changes of geometry and afterload in the pulmonary artery, resulting in increased PR. It is not clear which changes occur in the other pulmonary artery and $\mathrm{RV}$ to compensate for unilateral branch PS in a long-term scale and whether this compensation would make PR more aggravated or reduced. Also, PR fraction may be changed in respect to which side of the pulmonary artery was occluded because PR fraction was different in individual branch pulmonary arteries after TOF repair. ${ }^{14}$ Finally, the influence of branch PS cannot be generalized to that of residual PS at other sites. This study excluded patients with significant branch PS that may cause pulmonary arterial geometric change and effect on PR. However, RVOT obstruction including infundibular, valvular, and supravalvular PS would not make asymmetric distortion of the pulmonary artery, and it is not influenced by differential regurgitation from individual branch pulmonary arteries. Therefore, our study does not have this kind of concern.

For less RV volume overload in patients with residual PS, we need to focus more on the change of RV diastolic performance by pressure load than the decrease of PR. Previous studies have discussed that restrictive RV physiology, the condition of decreased RV diastolic compliance, limited the adverse effects of pulmonary incompetence resulting in smaller RV size, improved exercise performance, and lowered arrhythmia propensity. ${ }^{15-17}$ Although some MRI studies have disagreed with influences of restrictive physiology, ${ }^{18-21}$ these results for restrictive physiology suggested that RV diastolic compliance has an important role against RV dilatation and progressive failure from PR. In our study, there was no difference in frequency of the restrictive physiology of RV between the 2 groups. Furthermore, the restrictive physiology of the RV was not related to RV volume in univariate analysis. Therefore, its compounding effects can be ruled out. However, decreased RV diastolic compliance in the hypertrophied RV caused by pressure overload can limit PR and prevent 

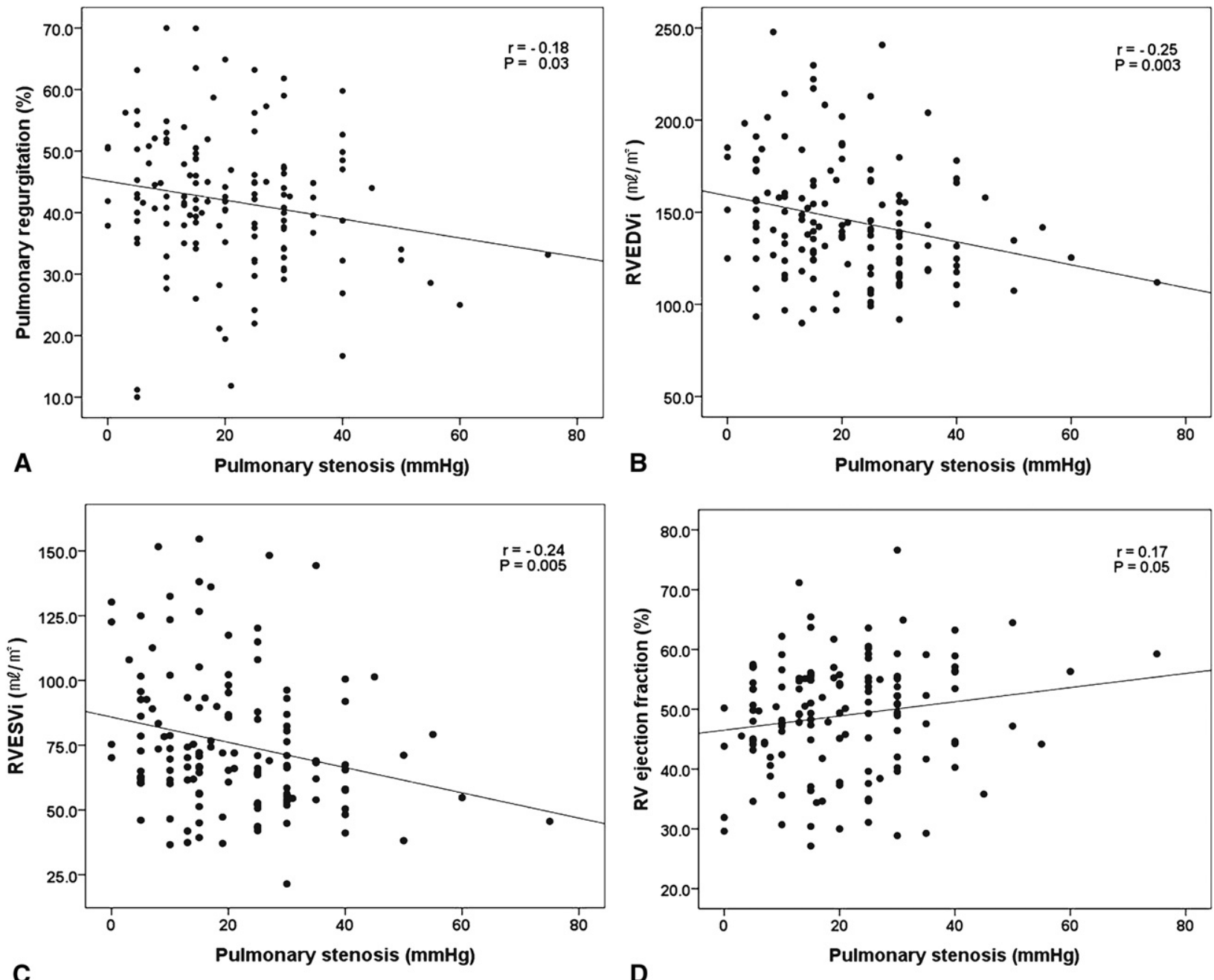

FIGURE 2. Impact of pressure load on right ventricular volume and function. Correlations between the peak pressure gradient of pulmonary stenosis and (A) pulmonary regurgitation fraction (\%), (B) right ventricular end-diastolic volume indexes (RVEDVi), (C) right ventricular end-systolic volume indexes $(R V E S V i)$, and (D) right ventricular $(R V)$ ejection fraction $(\%)$.

progression of RV dilatation with the similar mechanisms observed in the restrictive physiology.

Considering that severe RV hypertrophy caused by PS may have an adverse effect on RV function, it is difficult to determine an adequate degree of residual RVOT obstruction. In our study, the peak pressure gradient of PS in the PR with PS group was around 25 to $50 \mathrm{~mm} \mathrm{Hg}$ and the estimated RV pressure was 50 to $70 \mathrm{~mm} \mathrm{Hg}$, which was about half of LV pressure. These results suggest that a moderate degree of PS is harmless to RV function, being sufficient to prevent progressive RV dilatation. However, it is not enough to find an acceptable range of residual PS. Further conduction studies through cardiac catheterization are warranted to find an adequate degree of the RVOT

TABLE 3. Independent predictors for right ventricular volume and function

\begin{tabular}{|c|c|c|c|}
\hline Dependent variable & Predictor & $\beta$ & $P$ value \\
\hline \multirow[t]{2}{*}{ RV end-diastolic volume $\left(\mathrm{mL} / \mathrm{m}^{2}\right)$} & Pulmonary regurgitation $(\%)$ & 1.58 & $<.001$ \\
\hline & Peak pressure gradient of $\mathrm{PS}(\mathrm{mm} \mathrm{Hg})$ & -0.55 & .002 \\
\hline \multirow[t]{2}{*}{$\mathrm{RV}$ end-systolic volume $\left(\mathrm{mL} / \mathrm{m}^{2}\right)$} & Pulmonary regurgitation $(\%)$ & 1.04 & $<.001$ \\
\hline & Peak pressure gradient of $\mathrm{PS}(\mathrm{mm} \mathrm{Hg})$ & -0.49 & .005 \\
\hline \multirow[t]{2}{*}{ RV ejection fraction $(\%)$} & $\mathrm{RV}$ end-diastolic volume $\left(\mathrm{mL} / \mathrm{m}^{2}\right)$ & -0.13 & $<.001$ \\
\hline & $\mathrm{RV}$ end-systolic volume $\left(\mathrm{mL} / \mathrm{m}^{2}\right)$ & -0.28 & $<.001$ \\
\hline
\end{tabular}

$R V$, Right ventricular; $P S$, pulmonary stenosis. 
obstruction that could prevent massive RV dilatation without systolic deterioration.

About other factors which could affect RV mechanics, the PR with PS group had more previous palliative shunt histories that could have an adverse effect on RV dilatation. It is comprehensive that the patients who required palliative surgery owing to severe PS had more residual PS after total correction. In this respect, a recent study revealed that previous history of palliative shunt was not a risk factor for PVR. ${ }^{13}$ Furthermore, in patients of our study, the duration between shunt operation and total repair was short; therefore, its adverse effect, which could increase pulmonary vascular resistance, was not fully considered. In our study, both groups had many cases of transannular patch because of the previous policy of the surgery that allowed aggressive RVOT enlargement to prevent residual PS. This is different from our current policy, which does not use a transannular patch unless PS is very severe.

After the study, some patients in each group underwent pulmonary valve replacement. In the PR with PS group, the incidence of pulmonary valve replacement was less than that in the PR group without statistical significance (PR group, 36 [41.4\%]; PR with PS group, 17 [33.3\%]; $P=.32$ ). However, follow-up duration after the study was very short. Therefore, the influence of residual PS on the timing of pulmonary valve replacement cannot be assessed by these data. Further prospective study is needed.

\section{LIMITATIONS}

In our study, evaluating correlations between RV mass/ volume ratio and volume overload by measuring RV mass is considered to provide useful information for what changes prevent dilatation. However, we are unable to conduct such an evaluation because there is no measuring software of RV mass in our institute and manual measurement of RV mass may not be reliable. Furthermore, cardiac catheterization for conductance study is needed to evaluate whether changes occur in RV diastolic function under the pressure load.

\section{CONCLUSIONS}

From our study, it is suggested that a proper relief of RVOT obstruction with acceptable residual stenosis is more advantageous than aggressive RVOT enlargement in the long-term outcome of these patients with TOF repair. It could make $\mathrm{RV}$ diastolic property to protect against $\mathrm{RV}$ dilatation caused by PR. This result supports why we should choose minimal RVOT enlargement strategies despite residual PS.

Further follow-up studies involving cardiac MRI and cardiac catheterization are warranted to evaluate whether changes occur in the RV under the pressure load and to estimate the optimal range of residual PS, which prevents progressive RV dilatation without systolic deterioration.

\section{References}

1. Bouzas B, Kilner PJ, Gatzoulis MA. Pulmonary regurgitation: not a benign lesion. Eur Heart J. 2005;26:433-9.

2. Giardini A, Specchia S, Tacy TA, Coutsoumbas G, Gargiulo G, Donti A, et al. Usefulness of cardiopulmonary exercise to predict long-term prognosis in adults with repaired tetralogy of Fallot. Am J Cardiol. 2007;99:1462-7.

3. Gatzoulis MA, Balaji S, Webber SA, Siu SC, Hokanson JS, Poile C, et al. Risk factors for arrhythmia and sudden cardiac death late after repair of tetralogy of Fallot: a multicentre study. Lancet. 2000;356:975-81.

4. Discigil B, Dearani JA, Puga FJ, Schaff HV, Hagler DJ, Warnes CA, et al. Late pulmonary valve replacement after repair of tetralogy of Fallot. J Thorac Cardiovasc Surg. 2001;121:344-51.

5. Yetman AT, Lee KJ, Hamilton R, Morrow WR, McCrindle BW. Exercise capacity after repair of Tetralogy of Fallot in infancy. Am J Cardiol. 2001;87:1021-3. A5.

6. Ilbawi MN, Idriss FS, DeLeon SY, Muster AJ, Gidding SS, Berry TE, et al. Factors that exaggerate the deleterious effects of pulmonary insufficiency on the right ventricle after tetralogy repair: surgical implications. J Thorac Cardiovasc Surg. 1987;93:36-44.

7. Mulla N, Simpson P, Sullivan NM, Paridon SM. Determinants of aerobic capacity during exercise following complete repair of tetralogy of Fallot with a transannular patch. Pediatr Cardiol. 1997;18:350-6.

8. Davlouros PA, Kilner PJ, Hornung TS, Li W, Francis JM, Moon JC, et al. Right ventricular function in adults with repaired tetralogy of Fallot assessed with cardiovascular magnetic resonance imaging: detrimental role of right ventricular outflow aneurysms or akinesia and adverse right-to-left ventricular interaction. J Am Coll Cardiol. 2002;40:2044-52.

9. Geva T, Sandweiss BM, Gauvreau K, Lock JE, Powell AJ. Factors associated with impaired clinical status in long-term survivors of tetralogy of Fallot repair evaluated by magnetic resonance imaging. J Am Coll Cardiol. 2004;43:1068-74.

10. Puranik R, Tsang V, Lurz P, Muthurangu V, Offen S, Frigiola A, et al. Long-term importance of right ventricular outflow tract patch function in patients with pulmonary regurgitation. J Thorac Cardiovasc Surg. 4 November 2011 [Epub ahead of print].

11. Redington AN. Determinants and assessment of pulmonary regurgitation in tetralogy of Fallot: practice and pitfalls. Cardiol Clin. 2006;24:631-9.

12. Chaturvedi RR, Kilner PJ, White PA, Bishop A, Szwarc R, Redington AN. Increased airway pressure and simulated branch pulmonary artery stenosis increase pulmonary regurgitation after repair of tetralogy of Fallot. Real-time analysis with a conductance catheter technique. Circulation. 1997;95:643-9.

13. Kogon B, Plattner C, Kirshbom P, Kanter K, Leong T, Lyle T, et al. Risk factors for early pulmonary valve replacement after valve disruption in congenital pulmonary stenosis and tetralogy of Fallot. J Thorac Cardiovasc Surg. 2009;138:103-8.

14. Kang IS, Redington AN, Benson LN, Macgowan C, Valsangiacomo ER, Roman K, et al. Differential regurgitation in branch pulmonary arteries after repair of tetralogy of Fallot: a phase-contrast cine magnetic resonance study. Circulation. 2003;107:2938-43.

15. Cullen S, Shore D, Redington A. Characterization of right ventricular diastolic performance after complete repair of tetralogy of Fallot. Restrictive physiology predicts slow postoperative recovery. Circulation. 1995;91:1782-9.

16. Gatzoulis MA, Clark AL, Cullen S, Newman CG, Redington AN. Right ventricular diastolic function 15 to 35 years after repair of tetralogy of Fallot. Restrictive physiology predicts superior exercise performance. Circulation. 1995;91:1775-81.

17. Gatzoulis MA, Till JA, Somerville J, Redington AN. Mechanoelectrical interaction in tetralogy of Fallot. QRS prolongation relates to right ventricular size and predicts malignant ventricular arrhythmias and sudden death. Circulation. 1995; 92:231-7.

18. Sachdev MS, Bhagyavathy A, Varghese R, Coelho R, Kumar RS. Right ventricular diastolic function after repair of tetralogy of Fallot. Pediatr Cardiol. 2006; 27:250-5.

19. Eroglu AG, Sarioglu A, Sarioglu T. Right ventricular diastolic function after repair of tetralogy of Fallot: its relationship to the insertion of a "transannular" patch. Cardiol Young. 1999;9:384-91.

20. Helbing WA, Niezen RA, Le Cessie S, van der Geest RJ, Ottenkamp J, de Roos A. Right ventricular diastolic function in children with pulmonary regurgitation after repair of tetralogy of Fallot: volumetric evaluation by magnetic resonance velocity mapping. J Am Coll Cardiol. 1996;28:1827-35.

21. Apitz C, Latus H, Binder W, Uebing A, Seeger A, Bretschneider C, et al. Impact of restrictive physiology on intrinsic diastolic right ventricular function and lusitropy in children and adolescents after repair of tetralogy of Fallot. Heart. 2010; 96:1837-41. 